\title{
Effects of Dynamic and Isometric Vibration Exercises on Hemodynamics, Flexibility, and Stress Hormone Levels
}

\author{
BRITTANY ESPARZA, EUNICE DE LEON, AZENETH CHEVAILI, ENEIDA ALONSO, \\ MARGARITA GONZALEZ, MURAT KARABULUT
}

Health and Human Performance Lab; Department of Health and Human Performance; University of Texas Rio Grande Valley; Brownsville, TX

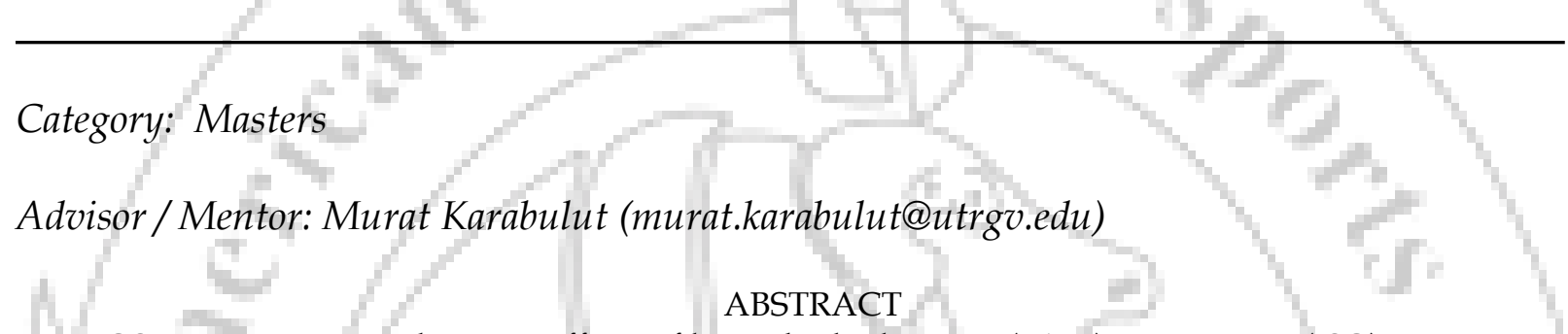

PURPOSE: To investigate the acute effects of lower body dynamic (DYN) vs isometric (ISO) exercises on systolic blood pressure (SBP) and diastolic blood pressure (DBP), heart rate (HR), flexibility using the sit and reach, and cortisol (COR) and amylase (AMY) levels measured pre and post exercise with different variations of frequency and amplitude on a power plate in pre-hypertensive females. METHODS: Nine females (age: $23.8 \pm 5.1 \mathrm{yrs}$ ) performed the ISO and DYN control sessions with the power plate off and ISO and DYN exercises with a combination of low frequency/high amplitude (LF/HA) and high frequency/low amplitude (HF/LA). A 5-min warm-up at $3.0 \mathrm{mph}$ on the treadmill occurred before testing. DYN exercises were performed from standing position to $120^{\circ}$ for squat, $90^{\circ}$ for lunge, and $90^{\circ}$ for squat for a total of four sets for one-min with metronome set at $40 \mathrm{bpm}$. ISO exercises were performed at the knee angles mentioned previously. Subjects had a $30 \mathrm{sec}$ rest in-between sets. Blood pressure (BP) and HR were recorded using an automated BP cuff pre, post, post $15-\mathrm{min}$, and post 30-min. Flexibility was recorded after the warm-up and post testing. The passive drool collection method was used to collect saliva to observe the changes in COR and AMY for a total of $1 \mathrm{~mL}$ for each session before the warm up and post-exercise. Vials were then placed in the freezer to await analysis. RESULTS: There was a significant condition*time interaction $(\mathrm{p}<.01)$ and time main effect $(\mathrm{p}<.01)$ for SBP in the LF/HA DYN condition; the decrease observed in SBP occurred from post-exercise to post 15-min. There was a significant time main effect $(p<.01)$ for HR from pre to post. A time main effect $(p<.05)$ was found for AMY from pre to post. There were no significant condition*time interactions or time main effects for DBP, COR or flexibility. CONCLUSIONS: The results indicate that conditions did not cause any significant changes in psychological or physical stress. The greatest decrease in SBP following DYN exercises with the LF/HA setting could be due to increased mechanoreceptor and/or metaboreceptor activity and/or higher nitric oxide release causing greater vasodilation. The data suggest that this combination of exercise and setting may help manage or improve BP. Since this was an acute study, future studies using this setting should examine the chronic effects on BP. 